\title{
LINFADENECTOMIA RETROPERITONEAL LUMBOAÓRTICA LAPAROSCÓPICA EN CÁNCER DE TESTÍCULO
}

\author{
Ivar Vidal-Mora' y Octavio A. Castillo',2,3. \\ 'Unidad de Urología Clínica Indisa. Santiago. Chile. \\ ${ }^{2}$ Facultad de Medicina. Universidad Andres Bello. \\ ${ }^{3}$ Facultad de Medicina. Universidad de Chile. Santiago. Chile.
}

\begin{abstract}
Resumen.- La Linfadenectomía Retroperitoneal Lumboaórtica (LALA) laparoscópica pareciera ofrecer la misma precisión diagnóstica en la estadificación y los resultados a largo plazo que su contraparte abierta. Además representa una opción viable en términos de morbilidad. Sin embargo son necesarios conocimientos laparoscópicos sólidos fundamentales para llevar a cabo esta cirugía en forma segura.
\end{abstract}

En la siguiente revisión abordaremos los principales aspectos de la técnica laparoscópica, incluyendo sus indicaciones, vías de acceso, técnica quirúrgica, y complicaciones, desarrollando además algunas controversias actuales sobre la linfadenectomía lumbo-aórtica laparoscópica por cáncer de testículo.
Palabras clave: Linfadenectomia retroperitoneal. Laparoscopía. Cáncer de testículo.

Summary.- Retroperitoneal Laparoscopic Lymph node Dissection (RPLND) seems to offer similar staging accuracy and long term outcomes to Open RPLND. It is also a reasonable option in terms of morbidity. However, solid laparoscopic skills are necessary to safely perform this surgery.

In the following article, we assess indications, access, surgical technique, complications and controversies of the laparoscopic RPLND.

Keywords: Retroperitoneal Lymph node dissection. Laparoscopy. Testis cancer.

\section{INTRODUCCIÓN}

La mejoría observada en las tasas de cura de los pacientes con cáncer de testículo, se basa en los esfuerzos conjuntos de las terapias médicas y quirúrgicas (1). A pesar de que la observación y la quimioterapia son opciones terapéuticas disponibles para los tumores testiculares de células germinales no seminomatosos (TCGNS), la linfadenectomia lumboaórtica abierta (LALA-A) continúa siendo el método más certero para la identificación de metástasis en los ganglios linfáticos. Sin embargo la invasividad y la morbilidad perioperatoria de este procedimiento son aspectos importantes a considerar y que han sido desarrollados por varios autores $(2,3)$.

La LALA laparoscópica (LALA-L) ha demostrado ser una alternativa posible con una baja tasa de complicaciones en manos experimentadas (4). Sin 
embargo todavía existen controversias respecto a la eficacia terapéutica respecto a la cirugía abierta para el manejo de TCGNS. A la vez los resultados oncológicos a corto y mediano plazo todavía son poco consistentes en la literatura.

En el siguiente artículo abordaremos los principales aspectos de la técnica, incluyendo sus indicaciones, vías de acceso, técnica quirúrgica, y complicaciones, desarrollando además algunas controversias actuales sobre la linfadenectomía lumboaórtica laparoscópica por cáncer de testículo y los principales resultados de nuestra serie.

\section{MÉTODOS}

Se realizó una revisión sistemática de literatura usando los términos, tumor de testículo, laparoscopía, linfadenectomía lumbo-aórtica, complicaciones, vigilancia y quimioterapia. Se utilizaron las bases de datos Medline, EMBASE y Cochrane. El criterio de inclusión fueron estudios controlados y randomizados o estudios observacionales de buena calidad.

Los objetivos primarios fueron sobrevida libre de enfermerdad y tasa de recaida. Los objetivos secundarios incluyeron morbilidad peri operatoria y complicaciones a largo plazo, clasificadas de acuerdo al sistema Clavien (5).

\section{Indicaciones de LALA laparoscópica}

Las indicaciones para LALA laparoscópica en la actualidad son las mismas que para cirugía abierta, vale decir:

1. Tumor testicular de células germinales no seminomatoso Etapa I.

2. Tumor testicular de células germinales no seminomatoso Etapa II con marcadores tumorales negativos.

3. Masa residual post quimioterapia con marcadores negativos.

4. Tumor testicular en etapa llb con marcadores negativos luego de un ciclo inicial abreviado de quimioterapia, independiente de la evolución de las lesiones en los estudios por imágenes.

En la evolución de la técnica, la indicación se reservó inicialmente para la estadificación de los tumores testiculares en etapa l, en candidatos ideales sin cirugía abdominal previa y de contextura dela- gada. Conforme se ha ido adquiriendo experiencia con la técnica laparoscópica, se asume que las indicaciones deben seguir la misma lógica que la cirugía abierta (6).

Recientemente Albaqmi y Janetscheck han publicado una serie de pacientes en quienes se ha indicado la lifadenectomia laparoscópica para monitorizar el éxito obtenido en el control de lesiones retroperitoneales $\mathrm{llb}$, tras un ciclo abreviado de quimioterapia (4).

\section{TÉCNICA QUIRÚRGICA}

\section{Diferentes tipos de templados modificados}

Todos los grupos laparoscópicos realizan una LALA modificada con resección en bloque del tejido linfático, siguiendo las recomendaciones de la cirugía abierta $(7,8)$.

Como ha sido demostrado la preponderancia temprana de enfermedad metastásica en el retroperitoneo para cáncer de testículo recae en la zona ipsilateral e infrahiliar. Nuestro grupo y los diferentes centros Europeos que realizan LALA laparoscópica, siguen los modelos propuestos por Weissbach y cols. (7), siendo la única modificación la inclusión de los nodos intercavoaórticos altos y precavos en el lado izquierdo.

Los centros norteamericanos, realizan la disección propuesta por Donohue y Foster (10). Recientemente, un centro en LALA laparoscópica publicó una disección amplia en el lado contralateral si se encuentran ganglios positivos (11).

\section{Preparación pre operatoria y posicionamiento}

Todos los pacientes recibieron la indicación de una dieta líquida el día previo a la cirugía. Un fleet enema también fue administrado previo a la hospitalización. Una vez que el paciente fue sometido a anestesia general fue adecuadamente posicionado, se instaló una sonda naso-gástrica y una sonda Foley. El paciente fue puesto en decúbito lateral izquierdo o derecho, dependiendo del lado indicado para la disección linfática.

\section{Procedimiento operatorio}

El pneumoperitoneo fue creado con una aguja de Veress y se fijó la presión intraabdominal en 15 $\mathrm{mmHg}$. 
Como ha sido demostrado la preponderancia temprana de enfermedad metastásica en el retroperitoneo para cáncer de testículo recae en la zona ipsilateral e infrahiliar (12). La extensión de la disección linfática en nuestra serie sigue las plantillas descritas por Weissbach y Boedefeld (13), siendo la única modificación la inclusión de los nodos intercavoaórticos altos y precavos en el lado izquierdo.

La preservación de las fibras nerviosas responsables de la eyaculación es la regla en todos los casos. Todos los casos fueron realizados con la intención de preservar los nervios y durante parte de nuestra experiencia nosotros no seccionamos los vasos lumbares para disecar el tejido bajo los grandes vasos, con la creencia que la diseminación linfática primaria en cáncer de testículo ocurre ventral a los vasos lumbares y que para procedimientos diagnósticos la exéresis de este tejido linfático no sería necesaria como ha sido propuesto por Holtl (14). Más recientemente estamos en el proceso de modificar nuestra técnica para realizar una cirugía con intención terapéutica.

\section{LALA L derecha}

El hilio renal derecho determina el límite superior de la disección y la marca anatómica para la sección inferior es el cruce del uréter con los vasos iliacos. Para comenzar la disección, el colon derecho es completamente movilizado, siendo el duodeno disecado a través de una maniobra de Kocher. La posición de flanco y la orientación de la mesa operatoria permiten que las vísceras caigan por fuera del campo quirúrgico.

Una vez que el retroperitoneo es expuesto, la arteria iliaca y el uréter son identificados, como también los vasos espermáticos, la vena cava, la aorta y el hilio renal. Desde caudal a cefálico, la disección comienza en la bifucarción de la iliaca y se dirige hacia arriba en sentido de la arteria renal derecha. El tejido linfático es incidido y disecado de la vena cava en su superficie anterior y bilateralmente. El tejido interaortocava es disecado cuidadosamente preservando las venas lumbares. El espécimen quirúrgico incluye los ganglios paracavos, precavos e interaortocavos.

\section{LALA L izquierda}

El colon izquierdo es desplazado medialmente. La disección se extiende de caudal a cefálico desde la arteria ilíaca izquierda, hasta los vasos renales izquierdos.

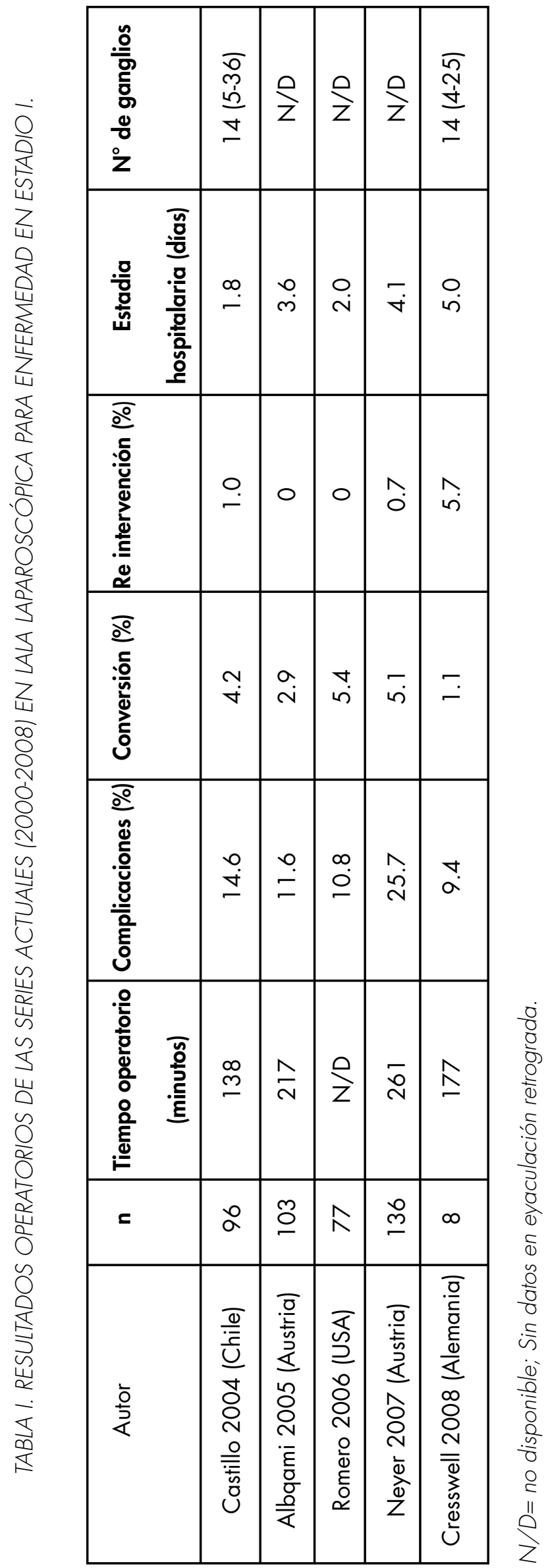


El espécimen quirúrgico incluye los ganglios pre aórticos y para aórticos y el tejido linfático interaortocava alto (ganglios localizados inferior a la vena renal izquierda)

Una movilización extensa del colon es esencial a cada lado como primer paso del procedimiento. Siempre realizamos la disección y resección del cordón espermático por debajo del área del anillo interno, siendo esto una parte fundamental de la técnica como describió Chang y cols (15). En nuestra serie hemos experimentado 3 metástasis del cordón y desde entonces prestamos especial interés a esta parte de la cirugía. Una vez que la cirugía está terminada extraemos el espécimen quirúrgico en una bolsa de polietileno.

\section{RESULTADOS}

\section{Evaluación de la LALA laparocópica}

\section{- Resultados operatorios}

En las series tempranas, la tasa de conversión varió entre un $5.8 \%$ y $13.3 \%$, debido fundamentalmente a sangrado incontrolable de grandes vasos, sin embargo en publicaciones recientes, la tasa de conversión cayó a 3.3\% (rango: 1.1-5.4\%; Tabla I) (16). El tiempo operatorio promedio de las series actuales disminuyó significativamente a 204 min. (rango:138-261 min). Inicialmente, la tasa de ganglios positivos vario entre un $5.8-62,5 \%$, pero en las últimas 6 series recientes, la tasa fue de $25 \%$ (1838; Tabla II) (16). Sólo 3 estudios contemporáneos registraron el número de ganglios positivos, con una media de 16 (14-20) (17-19).

\section{- Complicaciones}

Las tasas de complicaciones varía entre 5.6 y $46.7 \%$ en las series precoces, disminuyendo a $15.6 \%(9.4-25.7 \%)$ en las últimas series (16). Las complicaciones intraoperatorias mayores (Clavien III - IV), incluyen sangrado como también lesión ureteral, duodenal y de vesícula biliar. Con el incremento de la experiencia, la mayoría de las complicaciones pueden ser manejadas laparoscópicamente, lo que se refleja en la baja tasa de conversión $13.7 \%$; 1 $5.4 \%$ ) de las series recientes. Las complicaciones post operatorias tempranas fueron linfoceles, embolia pulmonar, mionecrosis, parestesia de extremidades inferiores, enfisema, una colitis pseudomembranosa e íleo paralítico. La mayoría de estas complicaciones se relacionaron a los largos tiempos operatorios relacionados a la curva de aprendizaje. Las complicaciones tardías consistieron en estenosis ureteral y eyaculación retrograda en $2-3 \%$, pero existen 3 reportes de metástasis en los puertos de trabajo $(0.3 \%)$

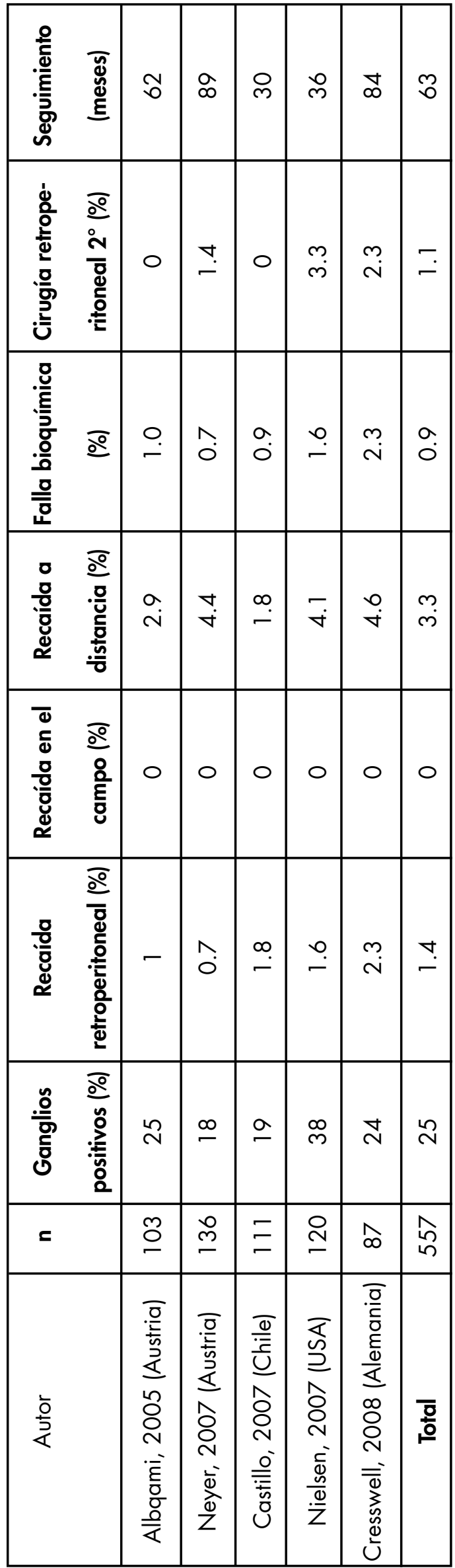


$(17,20,21)$. La tasa de re intervención en las últimas series fue de $1.4 \%(0.5-5.7 \%)$, incluyendo instalación temporal de un catéter doble-J o drenaje percutáneo de un linfocele (Tabla I). Debido a las diferencias en los sistemas de salud, no hay diferencias en las estadías hospitalarias entre las series iniciales y tardías. (1.2-6.4 días).

En nuestra serie se han presentado un 10 $\%$ complicaciones en pacientes en etapa I (17 pacientes). El tipo de complicación más frecuente es vascular y en nuestra experiencia estas pueden ser resueltas laparoscópicamente. En cuatro pacientes fue necesaria la conversión a cirugía abierta para resolver las lesiones. Registramos sólo tres pacientes con eyaculación retrograda. No registramos muerte perioperatoria.

\section{- Seguimiento oncológico}

Un 25\% de los 557 hombres de los 5 estudios con seguimiento a largo plazo, tuvieron ganglios positivos y 126 fueron sometidos quimioterapia adyuvante con 2 o 3 ciclos de la combinación de cisplatino, etopósido y bleomicina (BEP). La tasa de recaída en le retroperitoneo fue de $1.4 \%(0.7-2.3 \%)$, sin recurrencias en el campo, de acuerdo a los respectivos templados. Las metástasis a distancia (predominantemente pulmonares) ocurrieron en $3.3 \%$ (1.64.6) y la recurrencia bioquímica por si sola ocurrió en $0.9 \%(0-2.3 \%) .161$ pacientes $(29 \%)$ fueron tratados con quimioterapia, ya sea adyuvante $(n=126)$ o de salvataje $(n=35)$, resultando en una media de 0.6 ciclos (0.4-0.8). Seis de los 557 pacientes (1.1\%) requirieron remoción quirúrgica secundaria del tumor residual en el retroperitoneo, seguido de quimioterapia de salvación para la recaída retroperitoneal, revelando teratoma maduro en 4 pacientes y tejido necrótico en 2 pacientes. Después de un tiempo medio de observación de 63 meses (36-89 meses), todos los pacientes no tienen evidencia de enfermedad.

En nuestra serie tenemos un $77 \%$ de pacientes con linfonodos negativos. Con seguimiento medio de 25 meses (4-35). La tasa libre de recurrencia a 3 años para este grupo fue de $82 \%(95 \% \mathrm{Cl}: 64,91)$. El retroperitoneo fue el lugar de 5 recurrencias (1 asociada con el mediatino) y el pulmón en dos pacientes.

\section{DISCUSIÓN}

El manejo de los TCGNS en etapa I ha sufrido cambios significativos durante la última década. A pesar de que la LALA-A representa la primera opción para el diagnóstico y tratamiento de los TCGNS en etapa I, una estrategia adaptada al riesgo es la opción preferida, ya sea de observación o quimioterapia adyuvante $(22,23)$. La LALA-L ha sido recomendada como una alternativa que ofrece una estadificación ganglionar mínimamente invasiva, aumentando la seguridad que ofrece cualquier estrategia de vigilancia y evitando el sobretratamiento de la quimioterapia adyuvante (24-26). Sin embargo han surgido varias críticas: (1) plantillas insuficientes en la disección (2), técnica sin preservación de nervios, (3) procedimiento diagnóstico y no terapéutico y (4) la morbilidad de la curva aprendizaje.

¿Son las plantillas de la LALA-L lo suficientemente precisos como para que no haya necesidad de quimioterapia adyuvante cuando el estado de los ganglios es negativo? (8). Básicamente, esto corresponde a la incidencia de recidivas en el campo después de una LALA-L. En 557 pacientes, no se produjo recaídas en el campo, lo que refleja la exactitud diagnostica del procedimiento. Por otra parte, la tasa de metástasis ganglionares detectadas es en promedio de $25 \%$ tanto en los grupos abiertos como laparoscópicos. La tasa de recaída retroperitoneal fuera de las plantillas se observó en 1,2\% (rango: $0,7-2,3 \%$ ) de estas series. Cualquier recaída fuera de las plantillas puede indicar que se realiza una inadecuada disección de estos (8). Obviamente, la modificación de los templados pueden ser diferentes en Europa y los Estados Unidos, pero esto también se sucede en la LALA-A $(7,10)$.

Cualquier modificación de los templados representa un compromiso en la detección de ganglios positivos y en la preservación de eyaculación anterógrada. En este escenario, una tasa de recaída retroperitoneal de 1,4\% parece ser aceptable. La única modificación que nosotros hacemos a las plantillas clásicas de disección, es la extracción de los ganglios interaortocavos en cada disección. Esto aumenta la posibilidad de realizar una cirugía terapéutica. En cuanto a la disección bilateral, nosotros creemos que es posible y es nuestra tendencia hoy en día. Sin embargo el procedimiento debe ser ajustado de acuerdo al riesgo en particular de cada paciente y a los márgenes de sección fría de cada paciente.

¿Por qué realizamos nuestras cirugías con intención diagnóstica? Entendemos con intención diagnóstica en LALA a la realización de un procedimiento unilateral, sin evaluación de los márgenes de sección. La filosofía de esta aproximación es determinar la necesidad de tratamiento futuro. Esto se debe a que iniciamos nuestra experiencia hace 12 años y realizábamos disección unilateral sin evaluación de los márgenes de sección intraoperatorios. Por lo tanto nuestros pacientes recibieron 2 ciclos de quimioterapia para asegurar el control oncológico. 
En relación a las complicaciones, la única lesión que obliga a conversión es la injuria vascular y el sangrado incontrolable. Pero es importante notar que este parámetro depende de la experiencia de cada grupo en particular, recalcando que esta cirugía debe ser hecha en centros de referencia con amplia experiencia en cirugía laparoscópica.

\section{Impacto terapeútico de la LALA-L}

La preocupación más frecuente sobre la LALA-L se centra en el impacto terapéutico del procedimiento (evitar quimioterapia). Sobre la base del estudio randomizado y controlado de Williams et al (27), la quimioterapia adyuvante en el caso de estadío patológico II ha sido aplicada en Europa antes de la introducción de la LALA-L y representa una recomendación tipo A en las guías de la EAU (23). Los centros laparoscópicos, incluyendo el nuestro, adoptaron la misma estrategia, pero no como compensación de una insuficiencia diagnóstica o terapéutica. Los defensores de la LALA-A hacen hincapié en que la tasa de recaída en el caso de $\mathrm{N}+\sin$ quimioterapia adyuvante oscila entre un $30 \%$ a un $50 \%$ dependiendo del número y el patrón histológico de los ganglios linfáticos involucrados y la elevación de los marcadores antes de la LALA $(28,29)$.

Los centros laparoscópicos siempre han excluído a los pacientes con marcadores elevados después de la orquiectomía. Varios centros europeos también han utilizado terapéuticamente la LALA-A en la enfermedad de bajo volumen (es decir, etapa II, pN1). El impacto terapéutico de la LALA-L ha sido probado recientemente, en 10 pacientes con ganglios linfáticos de positivos, mostrando una tasa comparable de $20 \%$ (19). El concepto de LALA con intención curativa apunta a reducir la tasa y el número de ciclos de quimioterapia en la población tratada.

Podriamos resumir que la opción curativa de la LALA-L en enfermedad de bajo volumen etapa II $(\mathrm{pN}+)$ debe estudiarse con mayor detenimiento (estudio internacional), pero el valor de este concepto en el caso de etapa IIB sigue siendo incierta.

\section{CONCLUSIONES}

La LALA abierta es un procedimiento con una alta tasa de complicaciones. La LALA laparoscópica representa una opción viable a su contraparte abierta en términos de morbilidad. Conocimientos laparoscópicos sólidos son fundamentales para llevar a cabo esta cirugía en forma segura.
La LALA laparoscópica necesita ser validada como un procedimiento seguro en términos de morbilidad y control del cáncer.

\section{BIBLIOGRAFÍA Y LECTURAS RECOMENDADAS ( ${ }^{*}$ lectura de interés $y^{* *}$ lectura fundamental)}

1. Donohue JP, Thornhill JA, Foster RS: Primary retroperitoneal lymph node dissection in clinical stage A non-seminomatous germe cell testis cancer: review of the Indiana University experience 1965-1989. Br J Urol 1993; 71: 326-35.

*2. Yoon GH, Stein JP, Skinner DG:Retroperitoneal lymph node dissection in the treatment of low-stage nonseminomatous germ cell tumors of the testicle: an update. Urol Oncol. 2005; 23(3):168-77.

**3. Carver BS, Sheinfeld J: The current status of laparoscopic retroperitoneal lymph node dissection for non-seminomatous germ-cell tumors. Nat Clin Pract Urol 2005; 2(7):330-5.

*4. Janetscheck G and Albqami N: Laparoscopic retroperitoneal lymph-node dissection in the management of clinical stage I and II testicular cancer. J Endourol 2005; 19:683.

5. Clavien PA, Sanabria JR, Strasberg SM. Proposed classification of complications of surgery with examples of utility in cholecystectomy. Surgery 1992;111:518-26.

**6. Sotelo y cols. Trucos y secretos en cirugía laparoscópica urológica.

**7. Weissbach L, Boedefeld EA, Horstmann-Dubral B. Surgical treatment of stage-I non seminomatous germ cell testis tumor. Final results of a prospectivemulticenter trial 1982- 1987. Testicular Tumor Study Group. Eur Urol 1990;17: 97-106.

8. Eggener SE, Carver BS, Sharp DS, Motzer RJ, Bosl GJ, Sheinfield J. Incidence of disease outside modified retroperitoneal lymph node dissection templates in clinical stage I or IIA nonseminomatous germ cell testicular cancer. J Urol 2007;177:937-43.

*9. Donahue JP, Zachary JM, Maynard SM. Distribution of retroperitoneal lymph node metastases in non-seminomatous testicular cancer. J Urol 1982; 128: 315 .

10. Donohue JP, Foster RS. Retroperitoneal lymphadenectomy in staging and treatment. The development of nervesparing techniques. Urol Clin $\mathrm{N}$ Amer 1998;25:461-8.

*11. Allaf ME, Bhayani SB, Link R. Laparoscopic retroperitoneal lymph node dissection: duplication of the open technique. Urology 2005;65:575-7.

12. Donahue JP, Zachary JM, Maynard SM. Dsitribution of retroperitoneal lymph node metastases in non-seminomatous testicular cancer. J Urol 1982; 128: 315 . 
**13. Weissbach L, and Boedefeld EA: Localization of solitary and multiple metastases in stage II nonseminomatous testis tumor as basis for a modified staging lymph node dissection in stage I. J Urol 1987; 138: 77.

14. Höltl L, Peschel R, Knapp R. Primary lymphatic metastatic spread in testicular cancer occurs ventral to the lumbar vessels. Urology 2002; 59: 114.

15. Chang SS, Farivar Mohseni H, Leon A. Paracolic recurrence: The importance of wide excision of the spermatic cord at retroperitoneal lymph node dissection. J Urol 2002; 167: 94-96.

16. Rassweiler J, Scheitlin W, Heidenreich A, Laguna MP, Janetschek G. Laparoscopic Retroperitoneal Lymph Node Dissection: Does It Still Have a Role in the Management of Clinical Stage I Nonseminomatous Testis Cancer? A European Perspective. Eur Urol 2008;54:1004-9.

**17. Cresswell J, Scheitlin W, Teber D, Stock C, Lenz E, Rassweiler J. Laparoscopic retroperitoneal lymph node dissection combined with adjuvant chemotherapy for pathological stage II disease in nonseminomatous rem cell tumors: a 15-year experience. BJU Int 2008;102: 844-8.

Castillo OA, Alvarez JM, Vitagliano G, Rami-

*18. rez D, Diaz M, Sanchez-Salas R. Retroperitoneal laparoscopic lymphadenectomy for stage I nonseminomatous testicular cancer. Arch Esp Urol 2007;60:59-66.

*19. Nielsen ME, Lima G, Schaeffer EM. Oncologicy efficacy of laparoscopic RPLND in treatment of clinical stage I nonseminomatous germ cell testicular cancer. Urology

2007;70:1168-72

20. Castillo O, Urena R, Pinto I, Olivares R, Portalier P. Laparoscopic retroperitoneal lymph node dissection for stage I and II NSGCT. J Urol 2004;171(Suppl):247-78, abstract 933.

21. Sebe P, Nouri M, Haab F, Doublet JD, Gattegno B, Thibault P. Metastasis to trocar after lympha- denectomy with retroperitoneal laparoscopy. Prog Urol 2001;11:307-9.

**22. Krege S, Beyer J, Souchon R. European consensus conference on diagnosis and treatment of germ cell cancer: a report of the second meeting of the European Germ Cell Cancer Consensus Group (EGCCCG): Part I. Eur Urol 2008;53:478-96.

**23. Albers $\mathrm{P}$, Albrecht W, Algaba F. EAU Working Group on Testis Cancer. EAU guidelines on testicular cancer. Arnhem, the Netherlands: European Association of Urology; 2008.

24. Janetschek G, Reissigl A, Peschel R, Hobisch A, Bartsch G. Laparoscopic retroperitoneal lymphadenectomy for clinical stage I nonseminmatous testicular tumor. Urology 1994;44:382-91.

*25. Rassweiler JJ, Seemann O, Henkel TO, Stock C, Frede T, Alken P. Laparoscopic retroperitoneal lymph node dissection for nonseminomatous germ cell tumors: indications and limitations. J Urol 1996;156:1108-13.

26. Rassweiler JJ, Frede T, Lenz E, Seemann O, Alken P. Longterm experience with laparoscopic retroperitoneal lymph node dissection in the management of low-stage testis cancer. Eur Urol 2000;37:251-60.

27. Williams SD, Stablein DM, Einhorn LH. Immediate adjuvant treatment versus observation with treatment at relapse in pathological stage II testicular cancer. N Engl J Med 1987;317:1433-8.

*28. Stephenson AJ, Bosl GJ, Motzer J. Retroperitoneal lymph node dissection for nonseminomatous germ cell testicular cancer: impact of patient selection on outcome. J Clin Oncol 2005;23:27818.

29. Rabbani F, Sheinfield J, Farivar-Mohensi H. Low volume nodal metastases detected at retroperitoneal lymphadenectomy for testicular cancer: pattern and prognostic factors for relapse. J Clin Oncol 2001;17:2020-5. 